\title{
THE 283-DAY PERIODICITY IN THE O-C DIAGRAM OF BETA LYRAE
}

\author{
J. M. KREINER AND G. PAJDOSZ \\ Mt Suhora Observatory, Cracow Pedagogical University, Poland \\ sfkreine@cyf-kr.edu.pl
}

A detailed analysis of an extensive collection of primary visual, photographic and photoelectric minima of Beta Lyrae was performed. After removing the effect of the increase in orbital period we applied the Fourier analysis to the $\mathrm{O}-\mathrm{C}$ residuals. The most prominent periodicity is 283 days with amplitude about 0.05 days. It confirms the period found in the photometric light curves of Beta Lyrae [Van Hamme, Wilson and Guinan (1995)]. The 283-d quasi-variation of the period may be explained by assymetries of the light curve near the minima caused by some instabilities in disc and/or hot spot(s) brightness. 